\title{
Thin Ohmic or superconducting strip with an applied ac electric current
}

\author{
E. H. Brandt \\ Max-Planck-Institut für Metallforschung, D-70506 Stuttgart, Germany
}

(Dated: October 27, 2018)

\begin{abstract}
The complex impedance, currents, and electric and magnetic fields are calculated as functions of resistivity and frequency or London depth for a long thin strip with applied ac current. Both Ohmic and superconducting strips are considered. While the inductance per unit length of the strip depends on the strip length logarithmically, the sheet current, magnetic field, resistance, and magnetic susceptibility are independent of this length. It is found that the enhancement of resistance by the skin effect in thin Ohmic strips is much weaker (logarithmic) than in thick wires.

PACS numbers: $74.25 . \mathrm{Nf}, 73.25 .+\mathrm{i}, 74.78 . \mathrm{Bz}, 74.78 . \mathrm{Db}$
\end{abstract}

The distributions of the electric and magnetic fields inside and around a long wire carrying an applied ac current $I_{a}$ is a standard problem in electrodynamics when the wire has circular cross section [1]. In this case, the solutions of Maxwell equations away from the current contacts depend only on the radial coordinate $r$ and are easily obtained for both Ohmic wires with resistivity $\rho$ and superconducting wires in the Meissner state with London penetration depth $\lambda$. However, when the wire has a non-circular cross section, e.g., for flat strips, the problem becomes difficult. While the theory of thin strips in an applied ac field with no applied current is known since long time - it is simpler since the strip length drops out from all final results - apparently, no analytic or transparent numerical solutions are available for the even more important response of thin strips to applied ac current. This response is required, e.g., to understand SQUID systems [2] or to analyze the important experiments on temperature dependent magnetic field profiles of superconducting strips [3, 4]. In particular, the dependences of inductance, resistance, and field and current distributions on $\lambda, \rho$, ac frequency, and strip length, are of principal interest but are still unknown.

In this paper we settle this fundamental question and obtain these dependences for a current-carrying thin strip of width $2 a$, length $l \gg a$, and thickness $d \ll a$, filling the volume $|x| \leq a,|y| \leq d / 2,|z| \leq l$. In this basic case, the magnetic field around the strip depends only on the sheet current $J(x)=\int_{-d / 2}^{d / 2} j(x, y) d y$, which like the current density $j$ and total current $I_{a}$ flows along $z$. Interestingly, the inductance per unit length of a long strip depends on its length $l$ although the field and current distributions in the strip and its resistance and magnetic susceptibility are independent of $l$.

This strip example has the additional advantage that the nontrivial magnetic field distribution at the surface of the conductor can be measured by magneto-optics using flat indicator films or linear arrays of Hall probes [3, 4]. Note that with cylindrical wires there is no analog to such field profiles since the wire surface $r=a$ exhibits constant and material-independent field magnitude $I_{a} / 2 \pi a$.

To solve the strip problem with minimum effort we de- rive an equation of motion for the sheet current $J(x, t)$, which in general is driven by a time-dependent perpendicularly applied magnetic field $H_{a}(x, t) \| y$ and/or an electric current $I_{a}(t)$ applied at the ends of the strip, far away from the considered section. This method implicitly accounts for the complicated magnetic field around the strip, which thus does not have to be cut off or approximated as in other numerical methods. For strips with no applied current in a perpendicular $H_{a}(t)$, integral equations for $J(x, t)$ and $j(x, y, t)$ were obtained in Refs. 5 for thin and thick strips with linear or nonlinear resistivity, assuming zero London depth $\lambda=0$. But actually, from the linear results of Refs. 5 one can obtain the equations and results also for a superconductor strip in the Meissner state with arbitrary $\lambda$, namely, by replacing the Ohmic (real) resistivity $\rho$ by an imaginary value $\rho(\omega)=i \omega \mu_{0} \lambda^{2}$. The circular frequency $\omega$ then drops out from the final (static) results.

General equations for thin and thick strips with both applied $H_{a}$ and $I_{a}$ and with finite $\lambda$ were derived in Ref. 6 using the rather general voltage-current law valid inside superconductors with or without vortices,

$$
E=E_{v}(\mathbf{r}, j, \mathbf{B})+\mu_{0} \lambda^{2}(\mathbf{r}) \partial j / \partial t .
$$

In it $E_{v}(\mathbf{r}, j, \mathbf{B})=\rho_{v}(\mathbf{r}, j, \mathbf{B}) j$ is the electric field caused, e.g., by moving vortices, and $\mathbf{B}$ is the magnetic induction. The second (London) term describes the acceleration of the massive charge carriers (Cooper pairs) by the electric field. The flux-flow term $E_{v}$ can be linear or nonlinear, e.g., $\rho_{v}=\rho_{F F}=$ const. $B$ for free flux flow, or $\rho_{v} \propto|j|^{n-1}$ for thermally activated depinning of vortices, where $n \gg$ 1 is the flux-creep exponent.

For the particular case of a thin strip with applied current $I_{a}$ but no applied field $H_{a}$ (or with large dc background field), the sheet current has the symmetry $J(-x)=J(x)$. The equation for $J(x, t)$ then reads $[6]$

$$
\frac{\partial J(x, t)}{\partial t}=\mu_{0}^{-1} \int_{0}^{a} d x^{\prime} K\left(x, x^{\prime}\right)\left[E_{a}(t)-E_{v}\left(x^{\prime}, t\right)\right] .
$$

Here $E_{v}\left(x^{\prime}, t\right)=E_{v}\left(x^{\prime}, j, B\right)$ with $j=J\left(x^{\prime}, t\right) / d\left(x^{\prime}\right)$ and $B=\mu_{0} H\left(x^{\prime}, t\right)$ the magnetic induction in the strip. The 
spatially constant electric field $E_{a}(t)$ (along $z$, like $j$ and $J$ ) formally drives the currents; it will drop out later when the solution is expressed in terms of $I_{a}(t)$. Finally, the integral kernel $K\left(x, x^{\prime}\right)$ is the inverse of a kernel $K^{-1}\left(x, x^{\prime}\right)$ and is defined by

$$
\begin{array}{r}
\int_{0}^{a} d x^{\prime \prime} K\left(x, x^{\prime \prime}\right) K^{-1}\left(x^{\prime \prime}, x^{\prime}\right)=\delta\left(x-x^{\prime}\right), \\
K^{-1}\left(x, x^{\prime}\right)=\frac{1}{2 \pi} \ln \frac{l^{2}}{\left|x^{2}-x^{2}\right|}+\Lambda(x) \delta\left(x-x^{\prime}\right),
\end{array}
$$

with $\Lambda(x)=\lambda^{2}(x) / d(x)$ the effective magnetic penetration depth of a superconductor film with thickness $d<\lambda$. Note that Eqs. (2) and (3) apply also to inhomogeneous strips with $\lambda, \rho_{v}$, and $d$ depending on $|x|$ sufficiently smoothly (over length scale $\gg d$ ). This property can be used to simulate edge currents $\underline{3}$, 44 caused by a geometrical barrier [7, 8] for flux penetration.

For a strip with Ohmic resistivity $\rho$ and applied ac current $I_{a}(t)=I_{a 0} \exp (i \omega t)$ one has $\Lambda=0$ and $E_{v}=$ $\rho_{v} j=\left(\rho_{v} / d\right) J$, or one may formally put $E_{v}=0$ and $\Lambda=$ $-i \rho /\left(\mu_{0} \omega d\right)$. Both methods yield the same equation for $J(x, t)=J_{0}(x) \exp (i \omega t)$ or $E(x, t)=\rho J(x, t) / d$ driven by $E_{a}(t)=E_{a 0} \exp (i \omega t)$,

$$
J_{0}(x)=-\frac{i E_{a 0}}{\mu_{0} \omega} \int_{0}^{a} d x^{\prime} K\left(x, x^{\prime}\right) .
$$

The integral kernel $K\left(x, x^{\prime}\right)$, Eq. (3), is now complex due to the imaginary $\Lambda$. Dividing this by the current amplitude $I_{a 0}=2 \int_{0}^{a} d x J_{0}(x)$, the electric field amplitude $E_{a 0}$ drops out:

$$
\frac{J_{0}(x)}{I_{a 0}}=\int_{0}^{a} d x^{\prime} K\left(x, x^{\prime}\right) / 2 \int_{0}^{a} d x \int_{0}^{a} d x^{\prime} K\left(x, x^{\prime}\right) .
$$

The magnetic field caused by this sheet current is $\mathbf{H}(x, y, t)=\mathbf{H}_{0}(x, y) \exp (i \omega t)=\left(H_{x}, H_{y}\right)$ with

$$
\mathbf{H}_{0}(x, y)=\nabla \times \hat{\mathbf{z}} \int_{-a}^{a} d x^{\prime} \frac{J_{0}\left(x^{\prime}\right)}{4 \pi} \ln \frac{l^{2}}{\left(x-x^{\prime}\right)^{2}+y^{2}} .
$$

Interestingly, the profiles $J_{0}(x)$ and $\mathbf{H}_{0}(x, y)$ obtained numerically from Eqs.(4)-(6) do not depend on the strip length $l$, though the kernel $K\left(x, x^{\prime}\right)$ looks different for different ratios $l / a$. This may be understood by physical arguments, but to prove it mathematically appears to be difficult. See also the similar theory for a double strip 9]. However, the complex resistance or impedance $Z=$ $i \omega L+R$ per unit length does depend on $l$ logarithmically ( $R=$ real resistance, $L=$ real inductance). Noting that $E_{a} l$ is the voltage drop along the strip, one obtains

$$
\frac{Z}{l}=\frac{E_{a 0}}{I_{a 0}}=i \omega \mu_{0} / 2 \int_{0}^{a} d x \int_{0}^{a} d x^{\prime} K\left(x, x^{\prime}\right) .
$$

We evaluate the integrals (2)-(7) using unit length $a=$ 1 and introducing a grid $x_{i}\left(0<x_{i}<1, i=1,2, \ldots, N\right)$

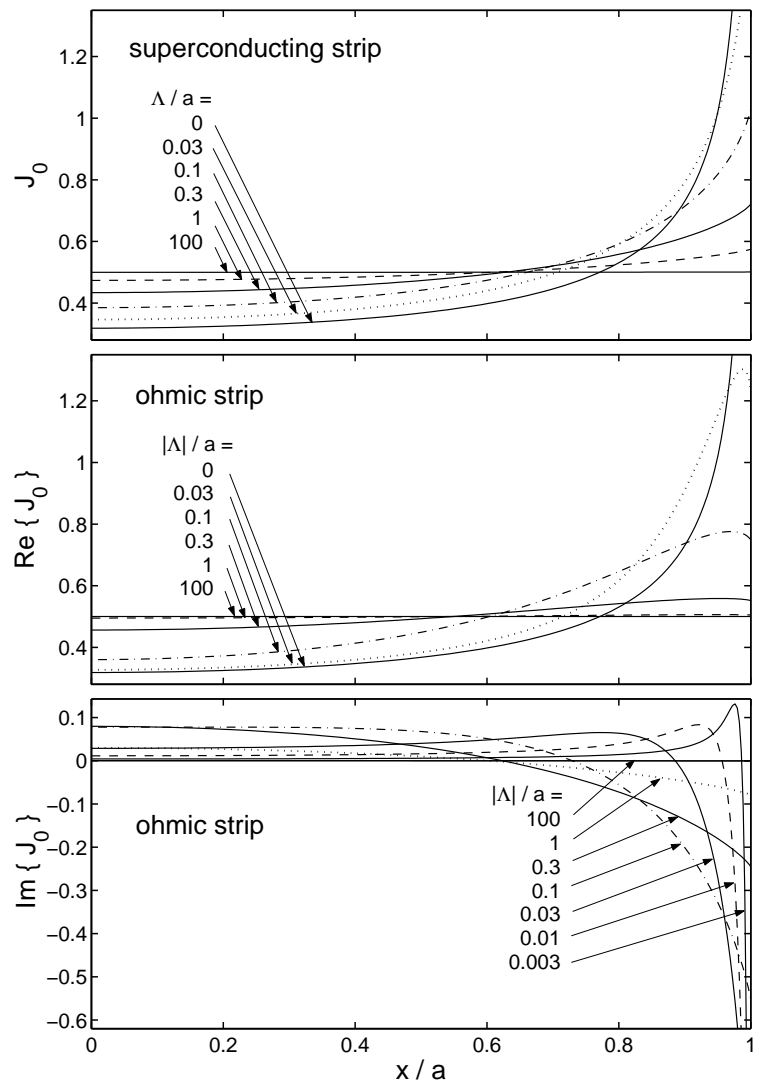

FIG. 1: The complex amplitude of the sheet current $J_{0}(x)$ in superconducting and Ohmic strips with applied ac current, Eq. (9). Shown are the real (in-phase) and imaginary (out-of-phase) components in units of $I_{a 0} / a$ for various $2 \mathrm{D}$ penetration depths $\Lambda$ or skin depth depths $\delta\left(|\Lambda|=\delta^{2} / 2 d\right)$.

with weights $w_{i}$ such that for any sufficiently smooth function $f(x)$ one has $\int_{0}^{a} f(x) d x \approx \sum_{i=1}^{N} w_{i} f\left(x_{i}\right)$. A grid with grid points densely spaced near the strip edge $x=1$, is obtained by introducing an auxiliary grid $u_{i}=\left(i-\frac{1}{2}\right) / N$ and then putting, e.g., $x_{i}=\left(3 u_{i}-u_{i}^{3}\right) / 2$, $w_{i}=3\left(1-u_{i}^{2}\right) / 2$. This amounts to a substitution of variables in the integral, $\int_{0}^{1} f(x) d x=\int_{0}^{1} f[x(u)] x^{\prime}(u) d u$. The integral kernel $K\left(x, x^{\prime}\right)$ now becomes a matrix [5, [6] :

$$
\begin{aligned}
K_{i j} & =\left(w_{j} Q_{i j}+\Lambda\left(x_{i}\right) \delta_{i j}\right)^{-1}, \\
Q_{i j} & =\frac{1}{2 \pi} \ln \frac{l^{2}}{\left|x_{i}^{2}-x_{j}^{2}\right|}, \quad i \neq j, \\
Q_{i i} & =\frac{1}{2 \pi} \ln \frac{\pi l^{2}}{x_{i} w_{i}},
\end{aligned}
$$

where $\delta_{i j}$ equals 1 when $i=j$ and 0 otherwise. The diagonal term $Q_{i i}$ in Eq. (8) is chosen such that the numerical error decreases with a high power of the grid number $N$, say, as $N^{-2}$ or $N^{-3}$, depending on the chosen grid, while any different choice gives a larger error $\propto N^{-1}$. The 


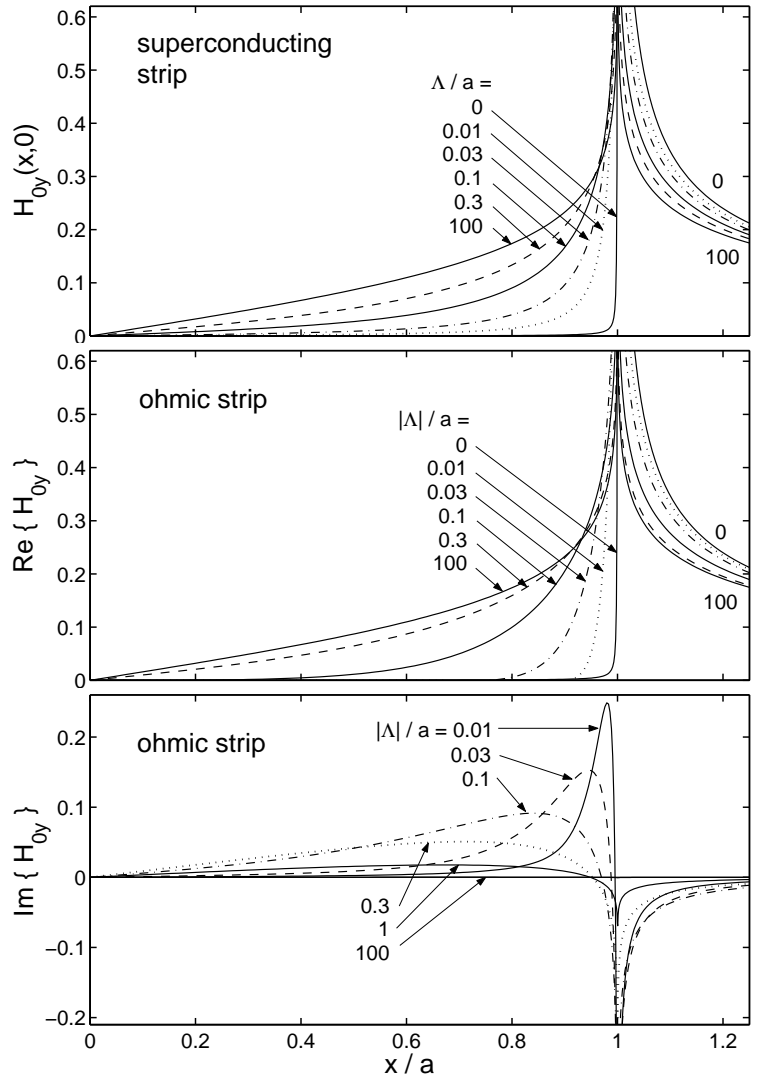

FIG. 2: The complex amplitude of the perpendicular magnetic field $H_{0 y}(x, 0)$ in the plane of superconducting and Ohmic strips with applied ac current, Eq. (10). Shown are the real and imaginary parts in units of $I_{a 0} / a$ for various $|\Lambda|$.

current and field profiles are then obtained as

$$
\begin{aligned}
J_{0}\left(x_{i}\right) & =\frac{I_{a 0}}{2 a} \sum_{j=1}^{N} K_{i j} / \sum_{k=1}^{N} \sum_{l=1}^{N} w_{k} K_{k l}, \\
H_{0 y}\left(x_{i}\right) & =\nabla_{i} \sum_{j=1}^{N} w_{j} Q_{i j} J_{0}\left(x_{j}\right),
\end{aligned}
$$

where $\nabla_{i}$ means the numerical derivative $d / d x$; this expression (10) is much more accurate than the direct computation of $H_{0 y}\left(x_{i}\right)$ from $J_{0}\left(x_{i}\right)$ by Ampère's Law using a matrix with singular terms $\propto 1 /\left(x_{i}-x_{j}\right)$.

The profiles $J_{0}(x)$ and $H_{0 y}(x, 0)$ for various $\Lambda$ are depicted in Figs. 1 and 2. For Ohmic and superconducting strips one has the same limits 10): at $|\Lambda| \ll a$ (ideal screening) one has $J_{0}(x)=\left(I_{a 0} / \pi\right)\left(a^{2}-\right.$ $\left.x^{2}\right)^{-1 / 2}, H_{0 y}(x, 0)=0$ for $|x|<a$ and $H_{0 y}(x, 0)=$ $\left(I_{a 0} / 2 \pi\right) \operatorname{sign}(x)\left(x^{2}-a^{2}\right)^{-1 / 2}$ for $|x|>a$, and at $|\Lambda| \gg a$ (weak screening) $J_{0}(x)=\left(I_{a 0} / 2 a\right)=$ const and $H_{0 y}(x, 0)=\left(I_{a 0} / 2 \pi\right) \operatorname{sign}(x) \ln |(a-|x|) /(a+|x|)|$. The imaginary (out-of-phase) components of $J_{0}(x)$ and $H_{0 y}(x, 0)$ are zero in these limits, but are maximum for Ohmic strips at some $x$ dependent value of $|\Lambda|=\delta^{2} / 2 d$ where $\delta$ is the skin depth.

The complex impedance, Eq. (7), is calculated as

$$
Z=i \omega L+R=i \omega \mu_{0} l / \sum_{k=1}^{N} \sum_{l=1}^{N} w_{k} K_{k l} .
$$

For superconducting strips this yields $R=0$ and the inductance (partly obtained analytically)

$$
L=\frac{\mu_{0} l}{2 \pi}\left[\ln \frac{2 l}{a}+\alpha\left(\frac{\Lambda}{a}\right)\right]+\frac{\mu_{0} l \Lambda}{2 a}
$$

with $\alpha(0)=0$ and $\alpha(\infty)=3 / 2-\ln 4=0.11371$. To good approximation, the first term is the geometric inductance $L_{m}$ and the last term the kinetic inductance $L_{k}$, while the small middle term $\propto \alpha$ is shared by both $L_{m}$ and $L_{k}$, which slightly depend on the current distribution, Fig. 1. For Ohmic strips we obtain $Z=i \omega L+R$,

$$
Z=i \omega \frac{\mu_{0} l}{2 \pi}\left[\ln \frac{2 l}{a}+\beta\left(\frac{|\Lambda|}{a}\right)\right]+R_{\text {strip }} \gamma\left(\frac{|\Lambda|}{a}\right)
$$

with $|\Lambda|=\rho /\left(\mu_{0} \omega d\right)=\delta^{2} / 2 d$ and $R_{\text {strip }}=\rho l / 2 a d$. The functions $\alpha, \beta$ and $\gamma$ are shown in Fig. 3. One has $\beta(0)=0$ (ideal screening), $\beta(\infty)=\alpha(\infty)=3 / 2-\ln 4$ (uniform current), $\gamma(\infty)=1$, and for $|\Lambda| \ll a, \gamma \approx$ $1+\left(2 / \pi^{2}\right) \ln (0.14 a /|\Lambda|)$ (skin effect). The same $\gamma$ results from Ohmic dissipation, $\gamma=4 a \int_{0}^{a}\left|J_{0}(x) / I_{a 0}\right|^{2} d x$, Eq. (5). Good fits for all $\Lambda$ are (see Fig. 3)

$$
\begin{aligned}
\alpha & \approx \alpha(\infty)(\tanh [0.435 \ln (\Lambda / 0.074 a)]+1) / 2 \\
\beta & \approx \alpha(\infty) \sqrt{(\tanh [0.92 \ln (|\Lambda| / 0.15 a)]+1) / 2} \\
\gamma & \approx 1+\left(4 / 3 \pi^{2}\right) \ln \left[1+(0.14 a /|\Lambda|)^{3 / 2}\right]
\end{aligned}
$$

For comparison we give here also the corresponding expressions for a uniform round wire of radius $a$, with applied current $I_{a}(t)=I_{a 0} \exp (i \omega t)$, current density $j(r, t)=j_{0}(r) \exp (i \omega t)$, and magnetic field $\mathbf{H}=$ $\hat{\varphi} H_{0}(r) \exp (i \omega t)$, where $\hat{\varphi}$ is the unit vector in azimuthal direction. Outside the wire $(r>a)$ one has simply $H_{0}(r)=I_{a 0} / 2 \pi r$, irrespective of the material properties and of $\omega$. Inside this wire $(r \leq a)$ one has

$$
\begin{aligned}
H_{0}(r) & =\frac{I_{a 0}}{2 \pi a} \frac{I_{1}(r / \lambda)}{I_{1}(a / \lambda)}=\lambda^{2} \frac{\partial j_{0}(r)}{\partial r}, \\
j_{0}(r) & =\frac{I_{a 0}}{\pi a^{2}} \frac{I_{0}(r / \lambda)}{I_{1}(a / \lambda)} \frac{a}{2 \lambda}=\frac{1}{r} \frac{\partial\left[r H_{0}(r)\right]}{\partial r},
\end{aligned}
$$

where $I_{0}(x)$ and $I_{1}(x)$ are Bessel functions. When $\lambda$ is real, Eqs. (17) and (18) are well known London solutions, and with imaginary $\lambda^{2}=\left(\rho / i \omega \mu_{0}\right)$, or complex $\lambda^{-1}=$ $(1+i)\left(\omega \mu_{0} / 2 \rho\right)^{1 / 2}=(1+i) / \delta$, they describe the skin effect in Ohmic wires with skin depth $\delta=\left(2 \rho / \omega \mu_{0}\right)^{1 / 2}$.

The complex resistance $Z=i \omega L+R$ and selfinductance $L$ of this cylindrical wire may be obtained from the energy balance [1]. In terms of real $I_{a}(t)$ and $E_{a}(t)$, the input power $I_{a} E_{a} l$ is the sum of the temporal change of the magnetic field energy outside the wire, 


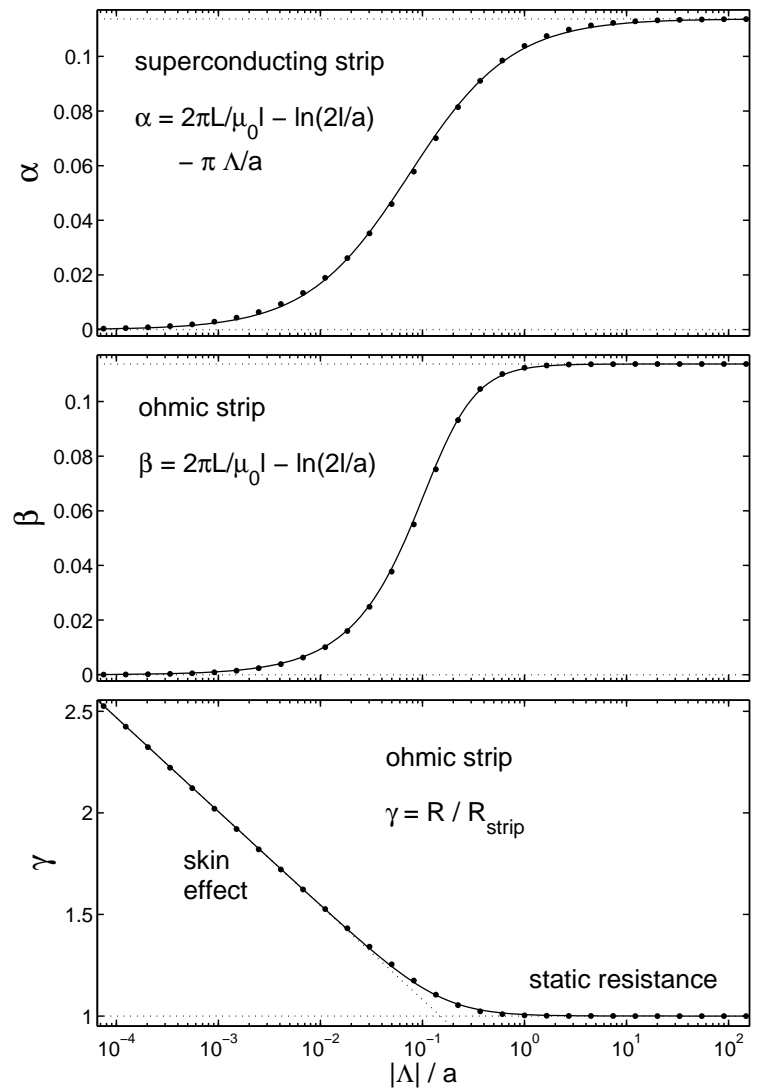

FIG. 3: The functions $\alpha, \beta$, and $\gamma$ of $|\Lambda| / a$ entering the inductance and resistance of a strip, Eqs. (12) and (13) (dots). The solid lines show the approximations Eqs. (14)-(16).

$(d / d t) \mu_{0} l \int_{a}^{r_{\text {out }}} 2 \pi r\left(I_{a} / 2 \pi r\right)^{2} d r=(d / d t) L_{e} I_{a}^{2} / 2$ defining the "external inductance"

$$
L_{e}=\frac{\mu_{0} l}{2 \pi} \ln \frac{r_{\text {out }}}{a} \approx \frac{\mu_{0} l}{2 \pi} \ln \frac{l}{a}
$$

with outer cut-off radius $r_{\text {out }} \approx l$, and the power dissipated or stored inside the wire (in the magnetic field and kinetic energy of the Cooper pairs). Using the Pointing vector, this power may be expressed in terms of the surface values $E_{s}=E(a, t)$ and $H_{s}=H(a, t)$, thus $I_{a} E_{a} l=(d / d t) L_{e} I_{a}^{2} / 2+H_{s} E_{s} \cdot 2 \pi a l$. Inserting here $H_{s}=I_{a} / 2 \pi a$ and dividing by $I_{a}$ we obtain

$$
E_{a}(t) l=L_{e} \dot{I}_{a}(t)+E_{s}(t) l .
$$

Writing the linear Eq. (20) in complex notation with $E_{a 0}=Z I_{a 0}, E_{s}=\rho j_{0}(a) \exp (i \omega t), j_{0}(a)$ from Eq. (18), and $R_{\text {wire }}=\rho l / \pi a^{2}$, we find

$$
Z=i \omega \frac{\mu_{0} l}{2 \pi} \ln \frac{l}{a}+R_{\text {wire }} \frac{a}{2 \lambda} \frac{I_{0}(a / \lambda)}{I_{1}(a / \lambda)} .
$$

Formula (21) applies both to superconducting wires with real London $\lambda$ (and $R_{\text {wire }}=i \omega \mu_{0} \lambda^{2} l / \pi a^{2}$ ) and to Ohmic wires with $\lambda=\delta /(1+i)$ making the second term complex. For superconducting wires with $\lambda \gg a$ this yields a kinetic inductance $L_{k}=\mu_{0} \lambda^{2} l / \pi a^{2}$. For Ohmic wires with large $\delta \gg a$, expansion of (21) adds a constant $\beta=1 / 4$ to $\ln (l / a)$ (from the "inner inductance") and a factor $\gamma=1+a^{4} / 48 \delta^{4} \approx 1$ to $R_{\text {wire. For small skin }}$ depth $\delta \ll a$, this constant is $\beta=\delta / a$ and the factor is $\gamma=R / R_{\text {wire }}=a / \delta \gg 1$, i.e., the resistance $R$ is strongly enhanced by the skin effect.

In conclusion, the dependences on $\Lambda, \rho, \omega$, and $l$, of the inductance $L$ and resistance $R$ of superconducting and Ohmic strips with applied ac current are obtained, Eqs. (12) to (16), and the current and magnetic field profiles are depicted. As compared to cylindrical wires, in strips the enhancement of $R$ by the skin effect is much weaker, $R / R_{\text {strip }}=\gamma \approx 1+0.2 \ln \left(0.3 a d / \delta^{2}\right)$ for small skin depth $\delta \ll a$ and $d<\delta$. Superconducting strips have a kinetic inductance $L_{k} \approx \mu_{0} l \Lambda / 2 a=\mu_{0} \lambda^{2} l / 2 a d$. The geometric inductance of both Ohmic and superconducting strips is similar to that of cylindrical wires; it is dominated by the length-dependent factor $\ln (2 l / a)$ that originates from the magnetic field energy outside the conductor and does not depend on material parameters. This factor $\ln (2 l / a)$ results from Eq. (7) without having to introduce an outer cut-off here. The strip length $l$ enters when the integral kernel $K\left(x, x^{\prime}\right)$, Eq. (3), is derived [6] by integration of the 3D Laplacian kernel $1 / 4 \pi\left|\mathbf{r}-\mathbf{r}^{\prime}\right|$ over $z^{\prime}$, assuming $z$ independence of $J$. But, of course, the exact value of the cut-off length in the logarithm depends also on the return path of the current.

I thank J. R. Clem, G. P. Mikitik, and E. Zeldov for helpful discussions. This work was supported by the German Israeli Research Grant (GIF) No G-705-50.14/01.

[1] L. D. Landau and E. M. Lifshitz, Electrodynamics of Continuous Media (Pergamon, New York, 1960).

[2] D. Koelle, R. Kleiner, F. Ludwig, E. Dantsker, and J. Clarke, Rev. Mod. Phys. 71, 631 (1999).

[3] D. T. Fuchs, E. Zeldov, M. Rappaport, T. Tamegai, S. Oi, and H. Shtrikman, Nature 391, 373 (1998).

[4] D. T. Fuchs, E. Zeldov, T. Tamegai, S. Oi, M. Rappaport, and H. Shtrikman, Phys. Rev. Lett. 80, 4971 (1998).

[5] E. H. Brandt, Phys. Rev. B 49, 9024 (1994); Phys. Rev. B 54, 4246 (1996).

[6] E. H. Brandt, Phys. Rev. B 64, 024505 (2001).

[7] E. Zeldov, A. I. Larkin, V. B. Geshkenbein, M. Konczykowski, D. Majer, B. Khaikovich, V. M. Vinokur, and H. Shtrikman, Phys. Rev. Lett. 73, 1428 (1994); T. Schuster, M. V. Indenbom, H. Kuhn, E. H. Brandt, and M. Konczykowski, Phys. Rev. Lett. 73, 1424 (1994).

[8] L. Burlachkov, A. E. Koshelev, and V. M. Vinokur, Phys. Rev. B 54, 6750 (1996).

[9] J. R. Clem and E. H. Brandt, Phys. Rev. B 72, 174511 (2005).

[10] W. T. Norris, J. Phys. D 3, 489 (1970); E. H. Brandt and I. Indenbom, Phys. Rev. B 48, 12893 (1993); E. Zeldov, J. R. Clem, M. McElfresh, and M. Darwin, Phys. Rev. B 49, 9802 (1994). 